\title{
A Front-Back Confusion Metric in Horizontal Sound Localization: The FBC Score
}

\author{
Tim Fischer, Marco Caversaccio, Wilhelm Wimmer
}

\begin{abstract}
In sound localization experiments, currently used metrics for front-back confusion (FBC) analysis weight the occurring FBCs equally, regardless of their deviation from the cone of confusion. To overcome this limitation, we introduce the FBC Score. A sound localization experiment in the horizontal plane with 12 bilaterally implanted cochlear implants (CI) users and 12 normal hearing subjects was performed to validate the method with real clinical data. The overall FBC Rate of the CI users was twice as high as the FBC Score. For the control group, the FBC Rate was 4 times higher than the FBC Score. The results indicate that the FBC Rate is inflated by FBCs that show a considerable deviation from the corresponding value on the cone of confusion.
\end{abstract}

Keywords-Binaural cues, cone of confusion, cochlear implants, diagnostics in audiology, spatial hearing outcome measures

\section{INTRODUCTION}

$\mathbf{S}$ OUND source localization primarily relies on the evaluation of interaural time and level differences. Front-back confusions (FBCs) occur because sound sources on the cone of confusion are equidistant from the left ear and the right ear and thus provide identical interaural time and level differences for a listener [1]. An illustration for a sound source on the cone of confusion is given in Figure 1.

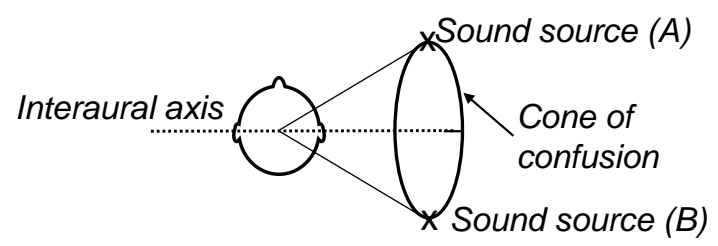

Fig. 1. The cone of confusion is defined as an imaginary cone extending outward from the center of the head [2]. The axis of the cone is defined by the interaural axis. Due to the symmetry of this cone around the interaural axis, sound source (A) and (B) produce identical interaural time and level differences as they are equidistant from the left ear and the right ear. Sound sources on the cone of confusion are thus useless for binaural sound localization.

FBC errors are not simply outliers with extreme error amplitudes. The underlying cause is different from normal localization errors (see Figure 1) which is why FBCs should be analyzed seperately [3]-[7].

There is a continuing debate in the literature on the definition of an FBC error. In horizontal sound localization experiments, the most common definition classifies responses crossing the interaural axis as FBCs [3]-[6], [8], [9]. This

T. Fischer, M. Caversaccio and W. Wimmer are with the Hearing Research Laboratory, ARTORG Center for Biomedical Engineering Research, University of Bern, Bern 3008, Switzerland and the Department of ENT, Head and Neck Surgery, Inselspital, Bern University Hospital, University of Bern, Bern 3008, Switzerland. E-mail address of the corresponding author: tim.fischer@artorg.unibe.ch. definition, which defines the FBC Rate, is sufficient for experiments that only require a coarse angular resolution of the test setup or feedback method [7], [10], [11]. However, such setups limit the measurement resolution for sound localization accuracy.

A refined $\mathrm{FBC}$ definition requires $\mathrm{FBC}$ responses to fall within a specific range of a response-dependent regression line, which is mirrored on the interaural axis [11], [12].

Further definitions as to what constitutes a FBC error are defined by fixed threshold values. These thresholds refer either to a minimum required deviation of the given response in relation to the interaural axis or to a maximum allowed deviation between the response and the stimulus mirrored on the interaural axis [13]. There is no general consensus regarding the magnitude of the thresholds.

Apart from thresholds that are not standardized, the current limitation of FBC analysis approaches is that all FBCs are considered equally strong. The deviation from the measurement position to which the error refers, which is the deviation of the response from the stimulus position mirrored on the interaural axis, is not considered. In addition, special care should be taken with stimuli presenting close to the interaural axis, as localization errors overlap with FBC errors in this area.

Herein, we propose a metric that allows to quantify the impact of FBCs on sound localization outcomes in the horizontal plane more precise than with the commonly used FBC Rate. The FBC Score can be applied regardless of the number of available localization results, the localization performance of the subjects and the measurement setup.

\section{Materials AND Methods}

\section{A. Study design and participants}

This study was designed in accordance with the Declaration of Helsinki and was approved by the local institutional review board (KEK-BE, No. 2018-00901). Twelve bilaterally implanted cochlear implants (CIs) users participated in the study, all using Sonnet (Med-El GmbH, Innsbruck, Austria) processors with an omnidirectional microphone setting. The CI users had a monosyllabic word recognition score in quiet of $70 \%$ or better at $60 / 65 \mathrm{~dB}_{\mathrm{SPL}}$. For comparison, a control group of 12 normal hearing $(\mathrm{NH})$ adults was included.

\section{B. Static Sound Source Localization Test}

Static sound source localization was performed with 12 equally spaced loudspeakers arranged in a circle around the subject. The test stimulus consisted of pink noise with $200 \mathrm{~ms}$ length. To prevent the use of monaural level cues, level roving between 60 to $70 \mathrm{~dB}$ SPL was applied. In total, 36 stimuli per 
subject were played, 3 stimuli per loudspeaker. The order of the stimuli with respect to the loudspeaker was randomized [10]. The loudspeakers were hidden behind a sound transparent curtain, no prior knowledge about possible stimuli directions was provided to the subjects. The subjects' feedback on the perceived location of the stimulus was recorded via a graphical user interface showing a dial with a resolution scale of 1degree angle and a login button.

\section{Calculation of the FBC Score}

We define the outcome of a sound localization experiment as a set of stimuli $\hat{\mathrm{S}}=\left\{\hat{\mathrm{s}}_{1}, \hat{\mathrm{s}}_{2}, \ldots, \hat{\mathrm{S}}_{\hat{\mathrm{N}}}\right\}$ and corresponding subject responses $\hat{\mathrm{R}}=\left\{\hat{\mathrm{r}}_{1}, \hat{\mathrm{r}}_{2}, \ldots, \hat{\mathrm{r}}_{\hat{\mathrm{N}}}\right\}$, where $\hat{\mathrm{N}}$ denotes the total number of test items. In relation to the FBC Rate (see Eq. 1), only responses that do cross the interaural axis are considered for the FBC analysis. After exclusion, we obtain a reduced set of stimuli $\mathrm{S}=\left\{\mathrm{s}_{1}, \mathrm{~s}_{2}, \ldots, \mathrm{s}_{\mathrm{N}}\right\}$ and responses $\mathrm{R}=\left\{\mathrm{r}_{1}, \mathrm{r}_{2}, \ldots, \mathrm{r}_{\mathrm{N}}\right\}$ with $\mathrm{N}$ items.

$$
\mathrm{FBC}_{\mathrm{RATE}}=\frac{\mathrm{N}}{\hat{\mathrm{N}}} \cdot 100
$$

The ideal position of an FBC, i.e., the position of a stimulus $\mathrm{s}_{\mathrm{i}}$ mirrored on the interaural axis, is defined as the FBC center $c_{i}$. We compute the deviation $\theta_{i}$ between the response $r_{i}$ and the $\mathrm{FBC}$ center $\mathrm{c}_{\mathrm{i}}$ as the shortest absolute angular difference (minor arc). An example for one stimulus-response pair is given in Figure 2. The maximum allowable deviation $\theta_{\max , \mathrm{i}}$ is defined by the interaural axis and the FBC center $c_{i}$ (see Figure 3 ). The farther away a response $r_{i}$ is from its corresponding FBC center and the closer to the interaural axis, the less likely it is to be an FBC. Therefore, we introduce a weighting factor $\mathrm{w}_{\mathrm{i}}$ for each measured FBC:

$$
\mathrm{w}_{i}=1-\frac{\theta_{i}}{\theta_{\max , i}}
$$

where $\theta_{\max , i}$ is the maximum deviation in the direction of the response (clockwise or counterclockwise) under consideration of the interaural axis. The weighting $\mathrm{w}_{i}$ ranges from 0 (response on the interaural axis) to 1 (a perfect FBC). An illustration of the procedure is given in Figure 3.

To provide a subject-level FBC metric that considers deviations of the responses from the cone of confusion and their proximity to the interaural axis, we propose the FBC Score as defined in Equation 3.

$$
\mathrm{FBC}_{\mathrm{SCORE}}=\mathrm{FBC}_{\mathrm{RATE}} \cdot \frac{1}{\mathrm{~N}} \sum_{\mathrm{i}=1}^{\mathrm{N}} \mathrm{w}_{\mathrm{i}}
$$

An FBC Score of $100 \%$ indicates that all responses were FBCs and exactly match the stimulus positions mirrored on the interaural axis. In contrast, an FBC Score of $0 \%$ would indicate that the responses did not contain any FBCs during the trial. For a calculation example with numeric data, a step-by-step guide for the calculation of the FBC Score with corresponding explanations is provided in the Appendix A. To facilitate the calculations, we implemented the procedure as a MATLAB function ${ }^{1}$. The script takes the sets of stimuli

\footnotetext{
${ }^{1}$ https://www.artorg.unibe.ch/research/hrl/data/fbc_score/
}

$\hat{\mathrm{S}}$ and responses $\hat{\mathrm{R}}$ as input parameters. In addition to the calculations described above, a separate analysis of front-back and back-front confusions is performed.

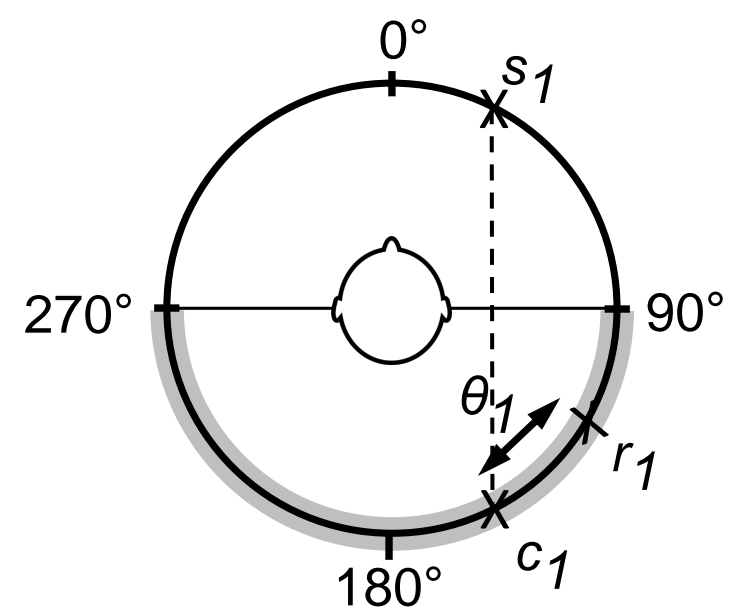

Fig. 2. The FBC center $c_{1}$ is the stimulus origin $\mathrm{s}_{1}$ mirrored on the interaural axis. The interaural axis corresponds to the line from $270^{\circ}$ to $90^{\circ}$. Valid FBCs can occur within the light shaded stripe. The deviation of the response $r_{1}$ from $c_{1}$ is denoted with $\theta_{1}$. In this example, the response $r_{1}$ lies in counter clockwise direction with respect to $\mathrm{c}_{1}$.

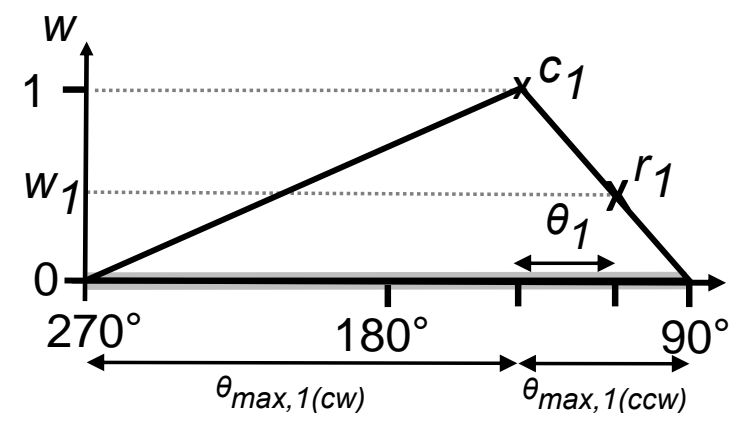

Fig. 3. Weighting factor calculation for the example shown in Figure 2. The $\mathrm{y}$-axis shows the front-back confusion weighting factor $\mathrm{w}$ and the $\mathrm{x}$-axis shows the rear azimuth. The deviation of the response $r_{1}$ from $c_{1}$ is denoted by $\theta_{1}$. The response $r_{1}$ lies in counter clockwise (ccw) direction with respect to $\mathrm{s}_{1}$, therefore $\theta_{\max , 1}$ is equal to $\theta_{\max , 1(\mathrm{ccw})}$. The areas $\theta_{\max , 1(\mathrm{ccw})}$ and $\theta_{\max , 1(\mathrm{cw})}$ are limited by the interaural axis as defined in Figure 2.

\section{Results}

For the CI users, the overall FBC Score is $27 \%$ in contrast to the almost twice as high FBC Rate of $47 \%$ ( $p<.001$, twosided Wilcoxon signed rank test). For the NH control group, the FBC Rate is 4 times as high as the FBC Score (FBC Score: $1 \%$, FBC Rate: $4 \%$; $p=0.062$ ). Table I shows the results of the FBC Score analysis compared to the FBC Rate for each $\mathrm{CI}$ user and $\mathrm{NH}$ subject.

\section{CONCLUSION AND Discussion}

For the evaluation of sound localization experiments, the errors are quantified in terms of their deviation from the stimulus position. However, for FBC analysis, thus far, only a rough distinction between "no $\mathrm{FBC}$ " and "FBC" is made, 
TABLE I

THE TABLE SHOWS THE RESULTS ON SUBJECT-LEVEL FOR THE FRONT-BACK CONFUSION (FBC) SCORE ANALYSIS COMPARED TO THE FBC RATE FOR EACH COCHLEAR IMPLANT (CI) USER AND NORMAL HEARING (NH) SUBJECT.

\begin{tabular}{lrr}
\hline ID & FBC Rate $(\%)$ & FBC Score $(\%)$ \\
\hline CI01 & 60.0 & 41.3 \\
CI02 & 46.7 & 19.8 \\
CI03 & 53.3 & 28.8 \\
CI04 & 50.0 & 39.9 \\
CI05 & 46.7 & 22.7 \\
CI06 & 43.3 & 26.9 \\
CI07 & 40.0 & 20.1 \\
CI08 & 46.7 & 18.2 \\
CI09 & 33.3 & 15.4 \\
CI10 & 50.0 & 31.3 \\
CI11 & 40.0 & 23.4 \\
CI12 & 50.0 & 27.8 \\
NH01 & 0.0 & 0.0 \\
NH02 & 0.0 & 0.0 \\
NH03 & 0.0 & 0.0 \\
NH04 & 0.0 & 0.0 \\
NH05 & 10.0 & 4.2 \\
NH06 & 6.7 & 0.0 \\
NH07 & 16.7 & 9.4 \\
NH08 & 0.0 & 0.0 \\
NH09 & 6.7 & 1.3 \\
NH10 & 0.0 & 0.0 \\
NH11 & 0.0 & 0.0 \\
NH12 & 3.3 & 0.0 \\
\hline
\end{tabular}

and the occurrences are counted. In this report, we propose a method for quantifying the severity of an $\mathrm{FBC}$, allowing a more precise analysis of this phenomenon.

In the example presented here, the FBC Rate would indicate that the tested subjects are prone to FBCs (47\%). However, the FBC Score shows that this assumption does not necessarily hold true since the impact of the FBCs is significantly smaller by a factor of $1.7(27 \%)$. Therefore, the FBC Rate in our example includes many FBCs with a considerable deviation from the corresponding stimulus position on the cone of confusion, to which this phenomenon actually refers.

An alternative option to illustrate this influence on the phenomenon of FBCs would be to indicate, in addition to the FBC Rate, the localization error with respect to the stimulus' position mirrored on the interaural axis. In our opinion, however, this approach may overestimate the importance of FBCs caused by stimuli near the interaural axis for two reasons: First, the influence of FBCs near the interaural axis is rather small in real life situations. Second, both error types, i.e., the FBC and localization errors, overlap for stimuli originating from close to the interaural axis, which can lead to distortions in the FBC analysis. By weighting the distance between the stimulus and the interaural axis and by considering the proximity of the stimulus to the interaural axis, this influence on the assessment of the FBC phenomenon can be mitigated. The effect of this weighting is particularly demonstrated by the 4 times lower FBC Score compared to the FBC Rate in the $\mathrm{NH}$ control group. Here, all FBC-causing stimuli originated from the measurement position with the smallest distance to the interaural axis.

The studies in [11], [12] defined responses as FBCs if the maximum deviation from the $\mathrm{FBC}$ center was less than or equal to a 40 or 45-degree angle. Such a static threshold value is not feasible in clinical studies with hearing-impaired subjects, due to the large differences in localization accuracy between the NH control and the test group [14]. An explanation of the underlying theoretical and psychoacoustic mechanism would be missing for the justification of such a threshold value. Besides the static threshold value, the iterative regression method applied in [11], [12] relies on the distribution and number of sound localization responses. The FBC Score does neither depend on a predefined threshold value nor on the quality or quantity of the localization results. This may be especially important when evaluating the performance of hearing-impaired patients in clinical trials. These localization results often show a wide individual variability [14] and consist of limited samples due to the limited testing time and subject attention.

\section{APPENDIX A}

\section{EXAMPLE CALCULATION WITH NUMERIC DATA}

In the following, we illustrate the calculation of the FBC Score using a small example data set with 5 stimuli $\hat{\mathrm{S}}$ and responses $\hat{\mathrm{R}}$ from a sound localization test in the horizontal plane (see Figure 4). Table II summarizes the results for the computational steps involved.

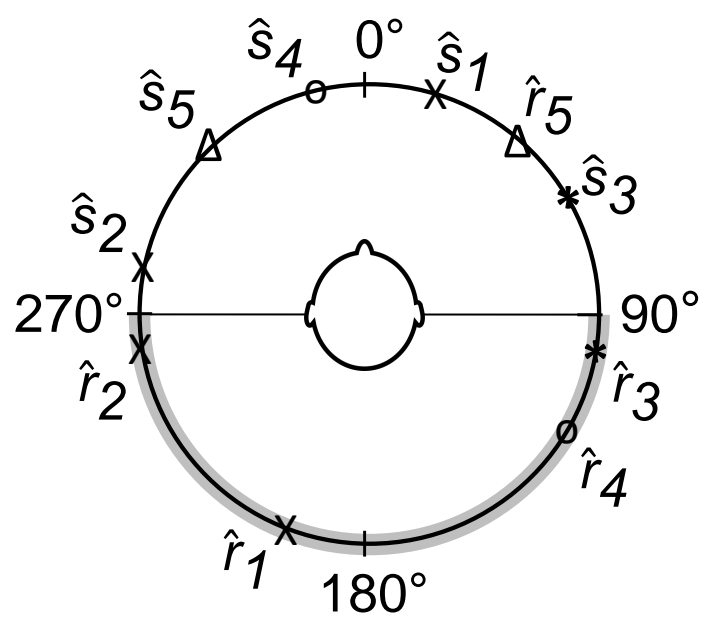

Fig. 4. Visualization of the data for the example calculation of the front-back confusion (FBC) Score. Stimuli are indicated with $\hat{\mathrm{S}}$ and responses with $\hat{\mathrm{R}}$. In this example, all stimuli lie inside the frontal azimuth and thus responses inside rear azimuth (light shaded area) are considered FBCs.

$$
\begin{aligned}
\hat{\mathrm{S}} & =\left\{\hat{\mathrm{s}}_{1}=20^{\circ}, \hat{\mathrm{s}}_{2}=280^{\circ}, \hat{\mathrm{s}}_{3}=65^{\circ}, \hat{\mathrm{s}}_{4}=350^{\circ}, \hat{\mathrm{s}}_{5}=315^{\circ}\right\} \\
\hat{\mathrm{R}} & =\left\{\hat{\mathrm{r}}_{1}=200^{\circ}, \hat{\mathrm{r}}_{3}=260^{\circ}, \hat{\mathrm{r}}_{3}=95^{\circ}, \hat{\mathrm{r}}_{4}=120^{\circ}, \hat{\mathrm{r}}_{5}=40^{\circ}\right\}
\end{aligned}
$$

Step 1 - Calculate the FBC Rate:

First, the FBC Rate is calculated. It is defined as the rate of the number of responses crossing the interaural axis with respect to the number of presented stimuli (see Eq. 1). Only $\hat{r}_{5}$ does not cross the interaural axis. Excluding $\hat{\mathrm{s}}_{5}$ and $\hat{\mathrm{r}}_{5}$ results in $\mathrm{N}=4$ pairs of stimuli $\mathrm{s}_{\mathrm{i}}$ and responses $\mathrm{r}_{\mathrm{i}}$ and an FBC Rate of $80 \%$. 
bioRxiv preprint doi: https://doi.org/10.1101/2020.02.12.945303; this version posted February 13,2020 . The copyright holder for this preprint (which was not certified by peer review) is the author/funder, who has granted bioRxiv a license to display the preprint in perpetuity. It is made available under aCC-BY-NC 4.0 International license.

TABLE II

DATA OF THE CALCULATION EXAMPLE.

\begin{tabular}{lllllll}
\hline $\begin{array}{l}\text { Test } \\
\text { item }\end{array}$ & $\begin{array}{l}\text { Stimulus }\left(^{\circ}\right) \\
\text { direction } \mathrm{s}_{\mathrm{i}}\end{array}$ & $\begin{array}{l}\text { Response }\left(^{\circ}\right) \\
\text { direction } \mathrm{r}_{\mathrm{i}}\end{array}$ & $\begin{array}{l}\mathrm{FBC} \text { center }\left(^{\circ}\right) \\
\mathrm{c}_{\mathrm{i}}\end{array}$ & $\begin{array}{l}\text { Deviation }\left(^{\circ}\right) \\
\theta_{\mathrm{i}}\end{array}$ & $\begin{array}{l}\text { Maximum }\left(^{\circ}\right) \\
\text { deviation } \theta_{\text {max }, \mathrm{i}}\end{array}$ & $\begin{array}{l}\text { Weighting factor } \\
\mathrm{w}_{\mathrm{i}}\end{array}$ \\
\hline 1 & 20 & 200 & 160 & 40 & 110 & 0.64 \\
2 & 280 & 260 & 260 & 0 & 10 & 1.00 \\
3 & 65 & 95 & 115 & 20 & 25 & 0.20 \\
4 & 350 & 120 & 190 & 70 & 100 & 0.30 \\
\hline
\end{tabular}

Step 2 - Calculation of $c_{i}$ and $\theta_{\mathrm{i}}$ :

The FBC center $c_{i}$ is obtained by mirroring the stimulus position $\mathrm{s}_{\mathrm{i}}$ on the interaural axis. The deviation $\theta_{\mathrm{i}}$ is calculated as the absolute difference between the response $r_{i}$ and $c_{i}$ measured over the minor arc. For example, for stimulus 3 , we have $\theta_{3}=\left|\mathrm{r}_{3}-\mathrm{c}_{3}\right|=\left|95^{\circ}-115^{\circ}\right|=20^{\circ}$.

Step 3 - Calculation of $\theta_{\text {max }, \mathrm{i}}$ :

The maximum deviation $\theta_{\max , i}$ between $r_{i}$ and $c_{i}$ is limited by the interaural axis. If $r_{i}$ lies in clockwise direction of $c_{i}$, the clockwise limit is applied $\left(\theta_{\max , \mathrm{i}}=\theta_{\max , \mathrm{i}(\mathrm{cw})}\right)$. The same applies for the counter clockwise direction.

For example, with stimulus $\mathrm{s}_{3}$, the minor arc for the response $\mathrm{r}_{3}$ lies in counter clockwise direction to the FBC center $c_{3}$, therefore the maximum deviation is $\theta_{\max , 3}=\theta_{\max , 3(\mathrm{ccw})}=25^{\circ}$.

Step 4 - Calculation of $\mathrm{w}_{\mathrm{i}}$ :

With $\theta_{\mathrm{i}}$ and $\theta_{\mathrm{max}, \mathrm{i}}$ we compute the weighting factor $\mathrm{w}_{\mathrm{i}}$ for each stimulus using equation (2).

Step 5 - FBC Score:

We now calculate the FBC Score as defined in Equation (3):

$$
\mathrm{FBC}_{\mathrm{SCORE}}=80 \% \cdot \frac{0.64+1.00+0.20+0.30}{4}=42.8 \%
$$

To facilitate the calculations, we implemented the procedure as a MATLAB function ${ }^{1}$.

\section{ACKNOWLEDGMENT}

The authors would like to thank Christoph Schmid from the Department of ENT, Head and Neck Surgery, Bern University Hospital (Inselspital), for the recruitment of the CI users of this study.

\section{REFERENCES}

[1] L. Rayleigh, "Xii. on our perception of sound direction," The London, Edinburgh, and Dublin Philosophical Magazine and Journal of Science, vol. 13, no. 74, pp. 214-232, 1907.

[2] H. Wallach, "On Sound Localization," J Acoust Soc Am, vol. 10, no. 1, pp. 83-83, 71938.

[3] S. Carlile, P. Leong, and S. Hyams, "The nature and distribution of errors in sound localization by human listeners," Hear Res, vol. 114, no. 1-2, pp. 179-196, 121997.

[4] F. L. Wightman and D. J. Kistler, "Resolution of front-back ambiguity in spatial hearing by listener and source movement," J Acoust Soc Am, vol. 105, no. 5, pp. 2841-2853, 51999.

[5] P. A. Hill, P. A. Nelson, O. Kirkeby, and H. Hamada, "Resolution of front-back confusion in virtual acoustic imaging systems," J Acoust Soc Am, vol. 108, no. 6, pp. 2901-2910, 122000.
[6] E. H. A. Langendijk, D. J. Kistler, and F. L. Wightman, "Sound localization in the presence of one or two distracters," J Acoust Soc Am, vol. 109, no. 5, pp. 2123-2134, 52001.

[7] M. T. Pastore, S. J. Natale, W. A. Yost, and M. Dorman, "Head Movements Allow Listeners Bilaterally Implanted With Cochlear Implants to Resolve Front-Back Confusions," Ear Hear, vol. 39, no. 6, pp. 12241231, 2018.

[8] P. Zahorik, P. Bangayan, V. Sundareswaran, K. Wang, and C. Tam, "Perceptual recalibration in human sound localization: Learning to remediate front-back reversals," J Acoust Soc Am, vol. 120, no. 1, pp. 343-359, 72006.

[9] Y. Cai, G. Chen, X. Zhong, G. Yu, H. Mo, J. Jiang, X. Chen, F. Zhao, and Y. Zheng, "Influence of Audiovisual Training on Horizontal Sound Localization and Its Related ERP Response," Front Hum Neurosci, vol. 12, p. 423, 102018.

[10] W. Wimmer, M. Kompis, C. Stieger, M. Caversaccio, and S. Weder, "Directional microphone contralateral routing of signals in cochlear implant users: A within-subjects comparison," Ear Hear., vol. 38, no. 3 , pp. 368-373, 2017.

[11] C. Montagne and Y. Zhou, "Audiovisual Interactions in Front and Rear Space," Front Psychol, vol. 9, 52018.

[12] E. A. Macpherson and J. C. Middlebrooks, "Localization of brief sounds: Effects of level and background noise," J Acoust Soc Am, vol. 108, no. 4, pp. 1834-1849, 102000.

[13] T. Letowski and S. Letowski, "Localization error: Accuracy and precision of auditory localization," in Advances in Sound Localization, P. Strumillo, Ed. Rijeka, Croatia: IntechOpen, 2011, ch. 4. [Online]. Available: https://doi.org/10.5772/15652

[14] M. A. Akeroyd, "An overview of the major phenomena of the localization of sound sources by normal-hearing, hearing-impaired, and aided listeners." Trends Hear., vol. 18, 122014. 